\title{
Validation of Already Reported SSR Molecular Markers Linked to White Rust Resistance Gene in Indian Mustard Brassica juncea
}

\author{
Chandana Behera ${ }^{*}$, D. K. Yadava ${ }^{2}$, Sujata Vasudev ${ }^{2}$, H. D. Pushpa ${ }^{3}$ and Naveen Singh ${ }^{2}$ \\ ${ }^{1}$ Department of Plant Breeding and Genetics, College of Agriculture, \\ Bhawanipatna, OUAT, India \\ ${ }^{2}$ Division of Genetics, ICAR-Indian Agricultural Research Institute, New Delhi-110 012, India \\ ${ }^{3}$ ICAR-Indian Institute of Oilseeds Research, Rajendranagar, Hyderabad- 500030, India \\ *Corresponding author
}

\begin{abstract}
A B S T R A C T
White rust caused by a fungus Albugo candida, is a destructive disease of many economically important species of Brassica, to control this resistance breeding is important .Marker validation is useful for testing reported markers in determining the target phenotypes in autonomous populations and dissimilar genetic backgrounds and also involves testing the reliability of marker to predict the phenotype, which defines whether or not a marker could be used in routine screening for MAS. The current study was carried out with the objective to validating the already reported intron polymorphic marker (At $5 \mathrm{~g}$ 41560 and At 5g 41940) from Arabidopsis at $1.2 \mathrm{cM}$ from AcB1-A4.1. 102 RIL population derived from Varuna $\mathrm{x}$ Bio YSR of Brassica juncea were validated along with their parents. Phenotypic and genotypic data of RIL population confirmed At5g41560 marker was polymorphic to both the parents as well as differentiate the resistance and susceptible individual of the population and found to be validated. But At5g41940 was found to be not validated. The present study indicated that At5g41560 marker could be used for screening the resistance and susceptible genotypes in oilseed breeding programs. For the introgression of white rust resistance gene, at $5 \mathrm{~g} 41560$ marker could be used in MAS as it was not specific to genotype.
\end{abstract}

\section{Introduction}

White rust caused by the fungus Albugo candida is one of the destructive diseases in many agronomically important Brassica species including Brassica juncea. The extent of yield losses caused by white rust depends on the severity and duration of combined infection. Both the reproductive and vegetative phases of the plant are affected by this fungal pathogen, results in the development of white pustules on cotyledons and abaxial surface of leaves. When the pathogen spread systematically, it causes severe malformation of the inflorescence resulting in staghead formation. The combined infection of leaves and inflorescences causes yield losses of 20-60\% (Barbetti, 1981; Lakra and Saharan, 1989). Physiological specialization in white rust 
pathogen is classified according to the specificity to different Brassica species, but it is still lacking in systematic characterization regarding the specificity. Till now at least 13 races of $A$. candida have been identified on the basis of their specificity to different crucifer species (Verma et al., 1999). These races at times, may not retain their species specificity and can also attack the related species, i.e., host specificity in this white rust pathogen is not an absolute adaptation to a particular species, especially when the races are from hosts sharing a common genome (Liu et al., 1996). Petrie (1988) and Rimmer et al., (2000) reported that, race 2 of Albugo candida predominantly infects $B$. juncea after the detailed study on screening accessions belonging to other Brassica species. In Brassica juncea germplasm, white rust disease resistance sources are available. Resistance to white rust pathogen is governed by a single dominant gene in the germplam of Brassica juncea (Vignesh et al., 2009, 2011).

A number of studies have shown that resistance to white rust in Brassica species is governed by simple Mendelian inheritance; for example, a single dominant gene in B. juncea controls resistance against race 2 (Tiwari et al., 1988; Rimmer and Buchwaldt 1995), and a single dominant resistance gene in $B$. rapa, against race 2A (Kole et al., 1996), three dominant genes in B. napus control resistance against race 7 has been reported (Fan et al., 1983; Liu et al., 1996). There is no breakthrough work has been made in the development of resistant genotypes of B. juncea. against Albugo candida in spite the availability of stable donors for white rust. This is because of the obligate nature of white rust pathogen, that cannot be maintained in the laboratory which cause the typical phenotyping of this trait. The expression of disease requires specific environmental conditions that are difficult to create under natural field condition for routine and large scale screening of breeding material through artificial inoculation. Mapping of genes has been reported for several important traits such as seed coat colour, fatty acid content, oil content, growth habit and resistance against diseases, including white rust (Snowdon and Friedt, 2004) in the genus Brassicas. However, availability of molecular markers tightly linked to the gene(s) of interest would enable indirect selection at seedling stage without the need for creation of artificial epiphytotic conditions. A number of studies has been done in mapping of genes for resistance to white rust in $B$. juncea by RAPD and RFLP markers (Prabhu et al., 1998; Cheung et al., 1998), PCR based cleaved amplified polymorphic sequence (CAPS) marker (Varshney et al., 2004). RFLP markers are more labour intensive, demand more DNA and are expensive, likewise the RAPD markers are not reproducible and are usually dominant hence these are not ideal for use in MAS. For the studies of population genetics, mapping and MAS Simple sequence repeats or microsatellite (SSR) markers are excellent, as they are highly polymorphic, codominant, require less amount of DNA, can be easily automated for high throughput screening, reproducible and highly transferable between populations (Gupta et al., 1999). MAS, where molecular markers linked to the gene of interest facilitate the indirect selection of the linked gene or genes in breeding populations, without need for disease screening. The efficiency of markers across population can be determine by the validation of already available markers reported in the same species, which is a necessary prerequisite for use of marker in crop improvement as well as their effective use in marker assisted selection (MAS). Validation of the already reported markers for white rust was also carried out to test the efficiency of the markers across population. 


\section{Materials and Methods}

\section{Plant materials}

In the present study, the experimental material used includes white rust resistant Bio YSR, developed by NRC Plant Biotechnology (INGR No. 04099), IARI, New Delhi is an indigenous Yellow seeded, high yield oil content line and a highly susceptible to white rust, a popular, widely cultivated, high yielding, stable cultivar, 'Varuna', of Indian mustard, [B. juncea (L.) Czern. and Coss.] which is suitable for late and early sowing.RIL population was developed by continuous selfing the $\mathrm{F}_{1}$ plants obtained from the cross Varuna $x$ Bio YSR to $F_{6}$ generation and was used for marker validation as well as identification of new molecular markers linked to white rust resistance gene(s). Approximately 102 RIL population were used for linkage analysis of the marker with putatively linked white rust resistance locus in Brassica juncea.

\section{Phenotypic evaluation of RIL for White rust}

102 RIL population were evaluated for the disease reaction spraying the zoospore of white rust pathogen. Inoculum was prepared by collecting the zoospore from the highly cultivated susceptible plant variety of Brassica juncea, Varuna. The zoospore were scraped from the infected leaves and kept in distill water at $8 \mathrm{C}$ for 4 hours to enhance the germination of fungal spores. The concentration of zoospore was adjusted to approximately $1 \times 10^{4}$ spores per $\mathrm{ml}$, then this was sprayed over the foliage with a hand atomizer until runoff and was repeated for three to four times. For disease development the RIL population was covered with PVC sheet, plot was frequently irrigated to keep favorable condition for the disease development. White rust was observed and scored (Williams 1985) using 0 to 9 rating scale. Cotyledons that showed no symptoms or small necrotic flecks on the adaxial surface without sporulation were scored as 0 or 1 and were considered resistant, whereas, those showing scattered or coalescing pustules on the abaxial or adaxial surfaces were scored as 7 or 9 and were considered susceptible. Intermediate scores (3 and 5)were rarely observed.

\section{Molecular analysis for genotyping}

The leaf sample of 102 RIL were collected along with the parents (Bio YSR and Varuna). DNA was isolated from the leaves of all the plants using the standard protocol of CTAB method (Doyle and Doyle, 1990). DNA was diluted to a final concentration of $25 \mathrm{ng} / \mu \mathrm{l}$ using TE $(10 \mathrm{mM}$ Tris- $\mathrm{HCl}$ and $1 \mathrm{mM}$ EDTA). Equal amount of DNA from 10 highly resistant RIL lines were pooled to constitute the resistant bulk (RB) and similarly DNA from 10 highly susceptible lines was pooled to get the susceptible bulk (SB) for carrying out the bulked segregant analysis (Michelmore et al., 1991). 509 different SSR primers derived from Brassica (www.brassica.info/resource/ markers/ssrexchange.php) and Arabidopsis were used for parental polymorphisms, out of these 27 polymorphic primers were identified. PCR was carried out at standard protocol to get the amplified product.

The amplified products were separated by electrophoresis on $2 \%$ agarose gels in 1' TAE buffer (0.04 M Tris-acetate, 0.01 M EDTA) for $4 \mathrm{~h}$ at $80 \mathrm{~V}$. To determine the size of the polymorphic fragments, a 100-bp ladder DNA marker (MBI Fermentas, Vilnius, Lithuania) was used as size standard. DNA fragments were visualized by staining the gel using ethidium bromide and photographed using the gel documentation unit. 


\section{Results and Discussion}

Various DNA marker systems have been used for mapping and tagging the genes of interest in $B$. juncea. Prior to their use in the marker assisted breeding, the first important thing is the validation of these markers in the potential parental lines. Once the markers for respective traits are validated they can be used effectively in practical plant breeding programme through MAS. Hence, the availability of molecular markers tightly linked to the gene(s) of interest would enable direct selection at seedling stage without exhaustive phenotyping for the target traits. MAS can help in precisely deploying such genes/QTLs in large number of diverse germplasm lines in short time span with assured result. Furthermore, the linkage drags can also be minimized through background selection. Marker validation involves testing the reliability of markers to predict phenotype. This indicates whether or not a marker could be used in routine screening for
MAS (Ogbonnaa et al., 2001; Sharp et al., 2001).

In the present investigation, validation of already reported SSR markers, At5g41940 and At5g41560 (Panjabi et al., 2010) was done in two genotypes viz. Varuna and Bio YSR, and further in RILs population derived from a cross Varuna $\times$ Bio YSR. Of these two SSR markers At5g41560 clearly differentiated Varuna and Bio YSR, whereas At5g41940 could not differentiate the parental lines (Plate 4.1a and plate 4.1b).

PCR product with SSR marker At5g41560 differentiated between resistance and susceptible parents, whereas, in Bio YSR a unique band was present at 420bp and 465bp but in Varuna the unique band was present at $445 \mathrm{bp}$. There was a difference of $25 \mathrm{bp}$ and $20 \mathrm{bp}$ in case of Varuna from the unique band amplified in Bio YSR at 420bp and 465bp respectively (Plate 4.1a.).

Plate.4.1 Validation of markers (a) At5g41560 and (b) At5g41940 for white rust resistance in Resistance parent B- BioYSR and Susceptible parent V- Varuna; M- 100bp ladder

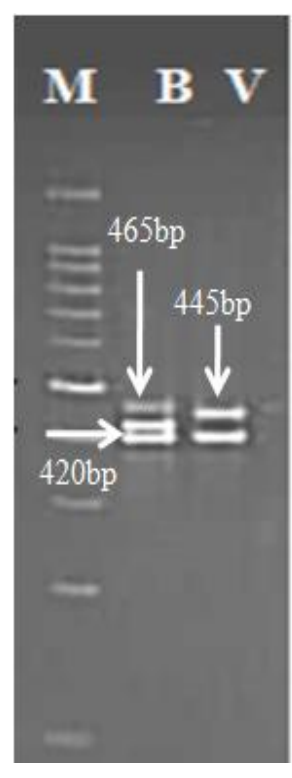

(a)

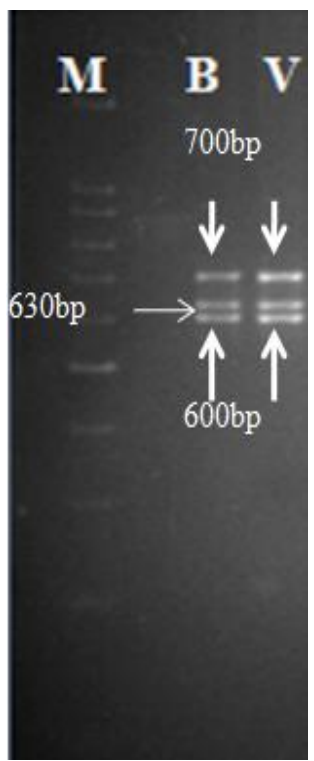

(b) 
Plate.4.2b Genotyping of 48 individual plants from the RIL population out of 102 from the cross Varuna $\times$ Bio YSR using the At5g41560 marker. Where M- DNA Marker lane, 100bp ladder, 148 individual plants of RILs population derived from cross Varuna $\times$ Bio YSR and *- represents the number of susceptible genotypes out of 48 genotypes
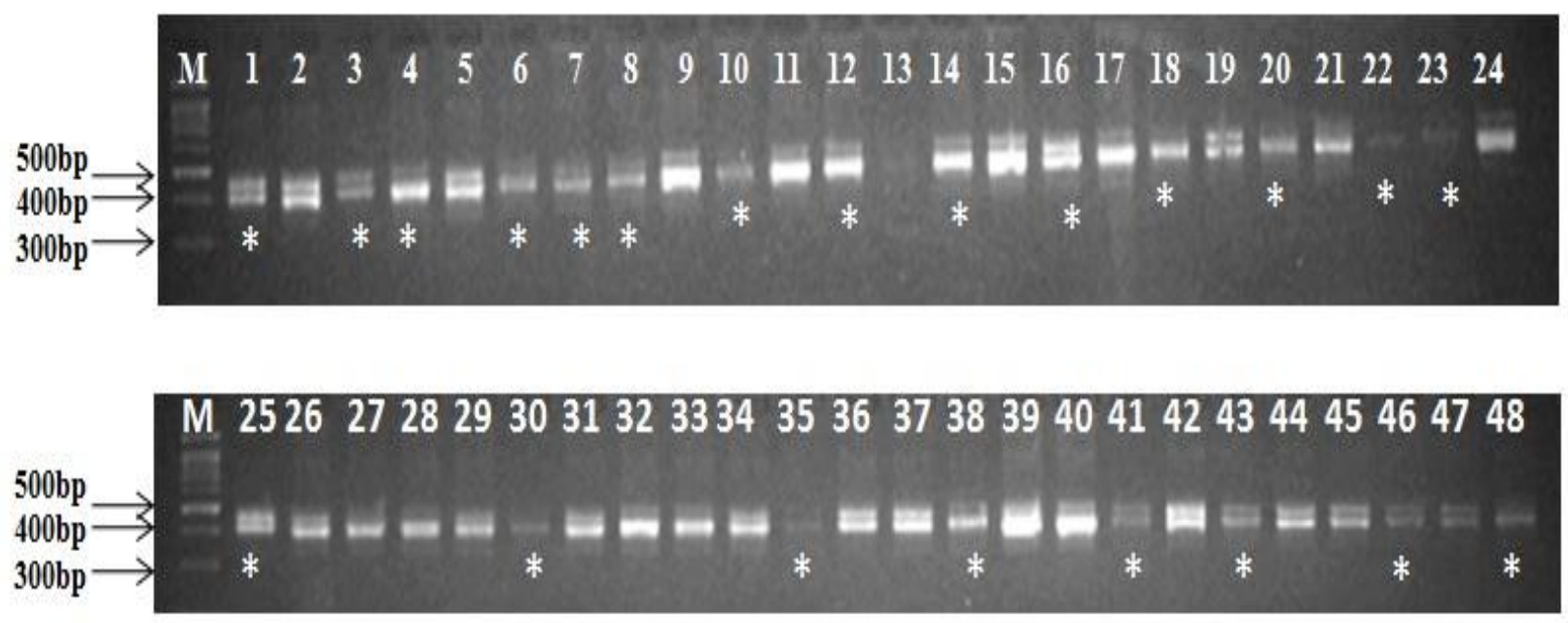

PCR Product with SSR marker At5g41940 could not differentiate resistant parent BioYSR from susceptible parent Varuna. There was a common band between these two parents 600bp, 630bp and 700bp. The genotyping of 102 RILs population was done with At5g41560 and At5g41940 markers. There was no polymorphism between the individual RILs plants with At5g41940 (Plate 4.2a). However, individual plants of RILs differentiated as resistant and susceptible genotype with At5g41560. The At5g41560 SSR marker segregated to give a genotypic ratio of $1: 1$. The segregation pattern in a set of 102 plants from the RIL generated from cross Varuna $\times$ Bio YSR is shown in plate $4.2 \mathrm{~b}$ which clearly indicates that the At5g41560 SSR marker differentiated between the resistant and susceptible genotypes.

Among these two reported markers only At5g41560 was validated as it gave the polymorphic bands in between resistance and susceptible parents. So At5g41560 could be used for the indirect selection of white rust resistance in a population through MAS.
In the present investigation, validation of already reported SSR markers viz., At5g41940 and At5g41560 (Panjabi et al., 2010) was done in two genotypes viz. Varuna and Bio YSR, and also in the RILs population derived from a cross Varuna $\times$ Bio YSR. SSR marker At5g41560 clearly differentiated Varuna and Bio YSR. However, the marker At5g41940 was amplified with the resistant donors studied, but it was not polymorphic between susceptible parent (Varuna) and resistance donor (Bio YSR).PCR product with SSR marker At5g41560 differentiated between resistance and susceptible parents, whereas, in Bio YSR a unique band was present at 420 bp and 465 bp but in Varuna the unique band was present at $445 \mathrm{bp}$. Additionally the marker At5g41560 which was $1.2 \mathrm{cM}$ away from AcB1-A4.1 locus also amplified in other resistant donor plants resulting in two amplicons of $420 \mathrm{bp}$ and 465 bp. So At5g41560 could be used for the indirect selection of white rust resistance in a population through MAS. Further studies are required to validate the already reported markers in different segregating population and also the validation of At5g41940 SSR 
markers in other mapping population. Marker validation in brassica has been reported for various traits (Pushpa et al., 2016) in B. juncea. Further the molecular markers are now used frequently for genetic diversity studies as well as for cross-transferability and polymorphic potential of different genomic markers in Brassica species (Kalita et al., 2007; Yadava et al., 2009; Vinu et al., 2013).

In conclusion the reported marker for white rust At5g41940 and At5g41560 (Panjabi et al., 2010) at the loci $1.2 \mathrm{cM}$ were validated in two genotypes Varuna and Bio YSR, and also in RILs population derived from a cross Varuna x Bio YSR. At 5g41560 clearly differentiated susceptible parent Varuna (unique band was at 445bp) and resistance parent Bio YSR (a unique band was at 420bp and 465bp) and found to be validated in two parents and revealed that this is not specific to the genotype. Whereas At5g41940 was unable to differentiate the parents, this is because of the specificity of the marker to a particular genotype.

\section{References}

Barbetti MJ (1981) Effects of sowing date and oospore seed contamination upon subsequent crop incidence of white rust (Albugo candida) in rapeseed. Aust. Plant Pathol 10: 44-46.

Berlin JD, Bowen CC (1964). The hostparasite interface of Albugo candida on Raphanus sativus. Am. J. Bot. 51: 445-452.

Cheung WY, Gugel RK, LandryBS (1998). Identification of RFLP markers linked to the white rust resistance gene $(\mathrm{Acr})$ in mustard (Brassica juncea (L.) Czern. and Coss.). Genome, 41: 626.628 .

Doyle JJ, Doyle JL (1990) Isolation of Plant DNA from fresh tissue. Focus 12: 1315.
Fan Z, Rimmer SR, Stefanson BR (1983) Inheritance of resistance to Albugo candida in rape (Brassica napus L). Canadian J Genet Cytol. 25: 420-424.

Gupta P K, Varshney R K, Sharma PC, Ramesh B (1999). Molecular markers and their application in wheat breeding: a review. Plant Breed. 118: 369-390.

Kole C, Teutonico R, Mengistu A, Williams PH, Osborn TC (1996) Molecular mapping of a locus controlling resistance to Albugo candida in Brassica rapa. Genetics 6: 367-369.

Lakra BS, Saharan GS (1989). Correlation of leaf and staghead infection intensities of white rust with yield and yield components of mustard. Indian $J$ Mycol Plant Pathol. 19: 279-281.

Lander ES, Green P, Abrahamson J, Barlow A, Daly MJ, Lincoln SE, Newburg L (1987). Mapmaker: an interactive computer package for constructing primary genetic linkage maps of experimental and natural population. Genomics 1: 174-181.

Liu JQ, Parks P, Rimmer SR (1996) Development of monogenic lines for resistance to Albugo candida from a Canadian Brassica napus cultivar. Phytopathol 86: 1000-1004.

Ogbonnaya FC, Subrahmanyam NC, Moullet O, de Majnik J, Eagles HA, Brown JS, Eastwood RF, Kollmorgen J, Appels R Lagudah ES (2001) Diagnostic DNA markers for cereal cyst nematode resistance in bread wheat. Aust J Agric Res., 52: 1367- 1374.

Panjabi P, Yadava SK, Sharma P, Kaur A, Kumar A, Arumugam N, Sodhi YS, Mukhopadhyay A, Gupta V, Pradhan AK, Pental D (2010) Molecular mapping reveals two independent loci conferring resistance to Albugo candida in the east European germplasm of oilseed mustard 
Brassica juncea. Theor Appl Genet, 121:137-145

PetrieGA (1988). Races of Albugo candida (white rust and staghead) on cultivated Cruciferae in Saskatchewan. Can J Plant Pathol 10: 142-150.

Prabhu KV, Somers DJ, Rakow G, GugelRK (1998). Molecular markers linked to white rust resistance in mustard Brassica juncea. Theor Appl Genet 97:865-870.

Rimmer SR, Mathur S, Wu CR (2000) Virulence of isolates of Albugo candida from western Canada to Brassica species.Can J PlantPathol22:229-235.

Rimmer SR, Buchwaldt L (1995) Diseases. In: Kimber, D. and McGreger, D. I. (eds), Brassica Oilseeds Production and Utilization, CAB Int., Oxford. pp. 111-140.

Snowdon RJ, Friedt W (2004) Molecular markers in Brassica oilseed breeding: current status and future possibilities. Plant Breeding 123: 1-8.

Tiwari AS, Petric GA, Downey RK (1988) Inheritance of resistance to $A$. candida race 2 in mustard (B. juncea $(\mathrm{L}$. Czern). Can J Plant Sci., 68: 297-300.

Verma PR, SaharanGS, Bartaria AM., Shivpuri A (1999) Biological races of Albugo candida on Brassica juncea and $B$. rapa var. toria in India. $J$ Mycol Pl Pathol 29: 75-82.

Vignesh M, Yadava DK, Sujata V, Yadav AK, Mohapatr T, Prabhu KV (2011)Characterization of an Indian mustard (Brassica juncea) indigenous germplasm line Bio-YSR for white rust resistance. Indian J. Plant Genet. Resour 24(1): 40-42.

Vignesh, M, Yadava DK, Sujata V, Mohapatra T, Jain N,Yadav AK, Malik D, Yadav MS, Prabhu KV (2009) Genetics of white rust resistance in Brassica juncea (L.) Czern. \& Coss. and allelic relationship between interspecific sources of resistance. Indian Journal of Genetics and Plant Breeding 69(3): 205-208.

Williams PH (1985) CRGC Resource Book. Deptt. of Plant Pathology, Univ. of Wisconsin, Madison, USA, pp. 1-7.

Varshney A, Mohapatra T, Sharma RP (2004). Development and validation of CAPS and AFLP markers for white rust resistance gene in Brassica juncea. Theor Appl Genet., 109: 153159.

Sharp PJ, Johnston S, Brown G, McIntosh RA, Pallotta M, Carter M, Bariana HS, Khatkar S, Lagudah ES, Singh RP, Khairallah M, Potter R, Jhones MGK (2001) Validation of molecular markers for wheat breeding. Australian Journal of Agricultural Research, 52: 1357-1366.

Kalita MC, Mohapatra T, Dhandapani A, Yadava DK, Srinivasan K, Mukherjee AK, Sharma RP (2007) Comparative evaluation of RAPD, ISSR and Anchored-SSR markers in the assessment of genetic diversity and fingerprinting of oilseed Brassica genotypes. Journal Plant Biochemistry and Biotechnology 16(1): 41-48.

Pushpa HD, Yadava DK, Singh N, Sujata V, Navinder S, Vignesh M, Prabhu KV (2016) Validation of molecular markers linked to low glucosinolate QTLs for marker assisted selection in Indian mustard (Brassica juncea L. Czern \& Coss). Indian J. Genet. 76(1): 64-68.

Vinu V, Singh N, Sujata V, Yadava DK, Kumar S, Naresh S, Bhat SR, Prabhu KV (2013) Assessment of genetic diversity in Brassica juncea (Brassicaceae) genotypes using phenotypic differences and SSR markers. International Journal of 
Tropical Biology and Conservation (Revista de Biología Tropical) 61(4): 1919-34.

Michelmore RW, Paran I, Kesseli RV (1991) Identification of markers linked to disease resistance genes by bulked segregant analysis: a rapid method to detect markers in specific genomic regions by using segregating populations. Proc Natl Acad Sci USA 88: 9828-9832.

\section{How to cite this article:}

Chandana Behera, D. K. Yadava, Sujata Vasudev, H. D. Pushpa and Naveen Singh. 2020. Validation of Already Reported SSR Molecular Markers Linked to White Rust Resistance Gene in Indian Mustard Brassica juncea. Int.J.Curr.Microbiol.App.Sci. 9(08): 1512-1519. doi: https://doi.org/10.20546/ijcmas.2020.908.175 